Review

\title{
Treatment of extensively drug-resistant Gram-negative infections in critically ill patients: Outcome of a consensus meeting at the 13th Asia-Pacific Congress of Clinical Microbiology and Infection, October 2012
} \author{
Taisheng $\mathrm{Li}^{\mathrm{k}}$, Xinjian Fan ${ }^{\mathrm{l}}$, Ursula Theuretzbacher ${ }^{\mathrm{m}, *}$ \\ a National University of Singapore, 1E Kent Ridge Road, Singapore 119228, Singapore \\ ${ }^{\mathrm{b}}$ Infectious Diseases Unit, Hospital Carlos G. Durand, Buenos Aires, Argentina \\ ${ }^{\mathrm{c}}$ First Department of Propaedeutic Medicine, Athens University School of Medicine, Athens, Greece \\ ${ }^{\mathrm{d}}$ Alfa Institute of Biomedical Sciences (AIBS), Athens, Greece \\ ${ }^{\mathrm{e}}$ Tufts University School of Medicine, Boston, MA, USA \\ ${ }^{\mathrm{f}}$ Department of Health Sciences, Clinical Pharmacology and Oncology Section, University of Florence, Florence, Italy \\ ${ }^{\mathrm{g}}$ Department of Medical Microbiology, Radboud University Nijmegen Medical Centre, Nijmegen, The Netherlands \\ ${ }^{\mathrm{h}}$ The First Hospital of China Medical University, Shenyang, China \\ ${ }^{\mathrm{i}}$ Huashan Hospital, Fudan University, 12 M. Wulumuqi Road, Shanghai 200040, China \\ ${ }^{\mathrm{j}}$ Department of Internal Medicine, National Cheng Kung University Medical College and Hospital, Taiwan \\ ${ }^{\mathrm{k}}$ Peking Union Medical College Hospital, Beijing, China \\ ${ }^{1}$ West China School of Medicine, West China Hospital, Sichuan University, Chengdu, China \\ ${ }^{\mathrm{m}}$ Center for Anti-Infective Agents, Eckpergasse 13, 1180 Vienna, Austria
}

Paul Anantharajah Tambyah ${ }^{\text {a }}$, Gabriel Levy Hara ${ }^{\mathrm{b}}$, George L. Daikos ${ }^{\mathrm{c}}$, Matthew E. Falagas ${ }^{\text {d,e }}$, Teresita Mazzei $^{\mathrm{f}}$, Johan W. Mouton ${ }^{\mathrm{g}}$, Andrea Novelli ${ }^{\mathrm{f}}$, Baiyi Chen ${ }^{\mathrm{h}}$, Minggui Wang ${ }^{\mathrm{i}}$, Wen-Chien Ko ${ }^{\mathrm{j}}$,

\section{A R T I C L E I N F O}

\section{Article history:}

Received 8 January 2013

Received in revised form 10 April 2013

Accepted 11 April 2013

\section{Keywords:}

Extensively drug-resistant

Gram-negative bacteria

Resistance

Carbapenems

Colistin

Aminoglycoside

Tigecycline

\begin{abstract}
A B S T R A C T
Infections caused by multidrug-resistant and extensively drug-resistant Gram-negative bacilli are increasingly challenging to manage in hospitals and long term-care facilities worldwide. As the therapeutic options are limited, the International Society of Chemotherapy in collaboration with the Asia-Pacific Society of Clinical Microbiology and Immunology organised a consensus conference as part of the 13th Asia-Pacific Congress of Clinical Microbiology and Infection. A panel of international experts from Europe, the Americas and Asia were convened to discuss the issues of therapeutic options for the management of these difficult-to-treat pathogens.
\end{abstract}

(C) 2013 International Society for Chemotherapy of Infection and Cancer. Published by Elsevier Ltd. All rights reserved.

\section{Contents}

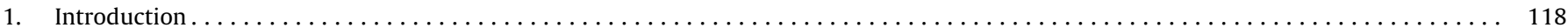

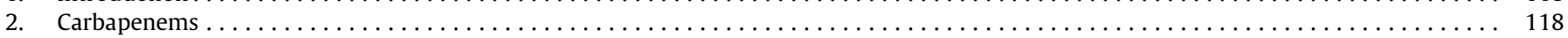

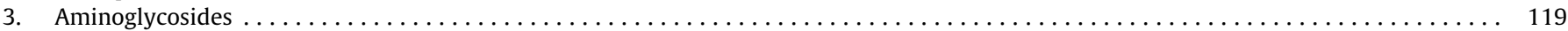

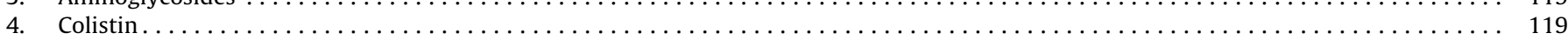

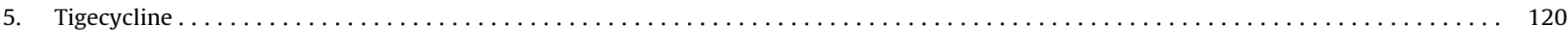

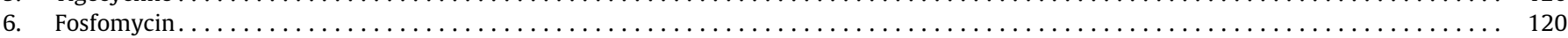

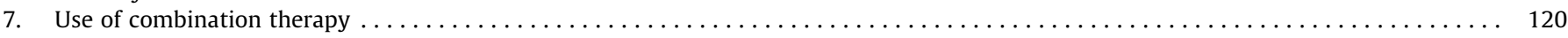

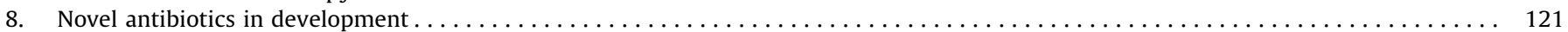

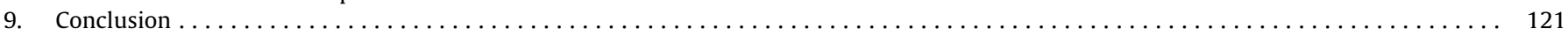

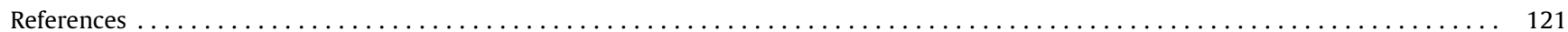

* Corresponding author. Tel.: +1 6504884890.

E-mail address: utheuretzbacher@cefaia.com (U. Theuretzbacher). 


\section{Introduction}

Worldwide antibacterial resistance is increasing dramatically [1]. The global magnitude of the resistance problem is difficult to assess. Conflicting definitions regarding combined resistances that are used by epidemiologists, microbiologists and clinicians have different implications for surveillance, public health and clinical case management. As the resistance problem continues to grow, harmonised definitions with which to describe and classify bacteria that are resistant to multiple antimicrobial agents are needed. The European Centre for Disease Prevention and Control (ECDC) and the US Centers for Disease Control and Prevention (CDC) published a standardised international terminology to facilitate grading various antimicrobial resistance profiles and reporting comparable data [2]. They define:

- multidrug-resistant (MDR) as organisms that are not susceptible to at least one agent in three or more classes of antibiotics to which they are usually susceptible (e.g. cephalosporins and $\beta$ lactamase inhibitor combinations, fluoroquinolones and aminoglycosides);

- extensively drug-resistant (XDR) as organisms that are susceptible only to one or two classes of antibiotics (mainly polymyxins and probably tigecycline); and

- pandrug-resistant (PDR) as organisms that are not susceptible to all known or licensed antibiotics currently available.

The scope of this discussion is confined mainly to organisms that would be thus classified as XDR, for which there are truly very few therapeutic options. Typical examples of clinically relevant and more prevalent XDR Gram-negative pathogens are Pseudomonas aeruginosa, Acinetobacter baumannii and carbapenemaseproducing Enterobacteriaceae (CPE) (mainly Klebsiella pneumoniae and Escherichia coli). Commonly, such XDR strains carry an extensive arsenal of unrelated resistance determinants such as multiple $\beta$-lactamase genes (classes A, B, C and D), 16S rRNA methylase ArmA- and RmtB-encoding genes (affecting all aminoglycosides) and dihydrofolate reductase genes (affecting trimethoprim), as well as changes in efflux pumps, chromosomally mediated porin modifications (affecting $\beta$-lactams and other compounds) and modified topoisomerases (affecting quinolones) [3]. The multidrug resistance pattern is the result of combined chromosome- and plasmid-encoded mechanisms. Selection may be caused by a wide range of unrelated antibacterial drugs and compounds.

Comparing worldwide epidemiological data is complicated when applying susceptibility/resistance categories defined by clinical breakpoint values. Such breakpoints may vary depending on the breakpoint-setting organisation. Although the major organisations such as the Clinical and Laboratory Standards Institute (CLSI) and the European Committee on Antimicrobial Susceptibility Testing (EUCAST) are trying to harmonise their recommendations, some discrepancies may remain partly related to the different specific purposes of the guidelines [4]. Both EUCAST and the CLSI issued breakpoint guidelines in 2011. These take into account certain mechanisms of resistance, wild-type population distributions and pharmacokinetic/pharmacodynamics (PK/PD) modelling [5,6]. A detailed discussion of the differences between the EUCAST and CLSI breakpoints is beyond the scope of this review but should be considered when comparing and defining multidrug resistance and extensive drug resistance.

Growing XDR rates among clinically important Gram-negative bacteria led to increasing usage of last-resort antibiotics, either as targeted therapy according to bacteriological susceptibility testing or even as empirical therapy in case of known high XDR rates in an institution. Giving a general and global recommendation for changing empirical choices in response to local XDR rates would not be feasible and would accelerate the emergence of resistance to last-resort antibiotics. Every institution should adapt their empirical regimens according to their own frequently updated microbiological findings and should carefully balance the decision with the consequence of increased selection pressure and potential emergence of resistance without remaining therapy options. Therefore, for the purposes of this manuscript we focus on targeted therapy options whilst bearing in mind that these recommendations can only be based on information garnered from observational studies, given the absence of randomised clinical trials. They need to be frequently adapted whenever new information becomes available.

\section{Carbapenems}

In general, the use of carbapenems needs to be weighed against the further selection of organisms that are intrinsically resistant to carbapenems such as Stenotrophomonas maltophilia or other important nosocomial pathogens such as meticillin-resistant Staphylococcus aureus (MRSA) or Candida spp. Moreover, even in the case of CPE (as discussed below), inappropriate usage of carbapenems could lead to the selection of increasingly resistant organisms in the unit or hospital. Although there is a great deal of reluctance to use carbapenems in the treatment of XDR organisms if the minimum inhibitory concentrations (MICs) are beyond the non-susceptible category, there may be a role for carbapenems in selected situations depending on the MIC and the choice of a combination partner. There is no clear evidence for the recommendation of carbapenems in such situations, but PK/PD considerations and preliminary clinical results point to the usefulness of carbapenems despite being in the non-susceptible category $[7,8]$. More clinical data are expected to become available in the near future.

Using available antibiotics in the setting of MDR and XDR infections highlights the importance of knowing the MIC value as it provides vital information beyond the breakpoint categories about the PD factor (MIC) in the PK/PD balance, especially in severely ill patients with high PK variability. Considering the MIC rather than the breakpoint category supports the decision for using a specific antibiotic as well as for choosing an optimised dosing regimen.

For carbapenems, although there are different MIC breakpoints as defined by EUCAST and CLSI, it may be possible to achieve a time above the MIC ( $T>$ MIC) for significant proportions of the dosing interval for organisms with MICs even up to $8 \mu \mathrm{g} / \mathrm{mL}$. Carbapenems display time-dependent bactericidal killing, and significant efficacy is obtained when free drug concentrations remain above the MIC for $40-50 \%$ of the time between dosing intervals. An optimised dosing regimen of a carbapenem (e.g. meropenem $2 \mathrm{~g}$ every $8 \mathrm{~h}$ or imipenem $1 \mathrm{~g}$ every $6 \mathrm{~h}$ ) with a prolonged infusion time of ca. 2-3 h each would increase the probability of attaining the PK/PD target. Meropenem $2 \mathrm{~g}$ every $8 \mathrm{~h}$ with a prolonged infusion time of ca. 2-3 h displays a relatively high probability (85\%) for bactericidal target attainment ( $50 \% T>$ MIC) when the MIC for the infecting organism is up to $8 \mu \mathrm{g} / \mathrm{mL}$ [9].

As the pharmacokinetics of the antibiotics cannot be reliably predicted in critically ill patients, it would be ideal to perform therapeutic drug monitoring (TDM) in these patients with XDR or PDR infections to ensure that the PD targets were indeed achieved [10]. Regrettably, in most countries, TDM is not routinely available for $\beta$-lactam agents such as carbapenems outside of research settings. For non-susceptible species with relatively low MICs, where a carbapenem is used in high doses despite the presence of a carbapenemase, it may be necessary to monitor concentrations achieved. On the other hand, if the MIC is $>8 \mu \mathrm{g} / \mathrm{mL}$, the risk of 
treatment failure increases with the MIC even when carbapenems are used in combination, so they should not be used as no substantial benefit can be expected based on the current evidence.

Beyond in vitro data and PK/PD modelling, there is scarce but growing data on the clinical use of carbapenems in the treatment of XDR and PDR organisms. Owing to the pressing clinical problem, more studies are being undertaken and results will hopefully be available soon. There is no evidence from direct head-to-head comparisons that meropenem, imipenem or doripenem is significantly better than any of the other comparators and thus no recommendation can be made for any of the three carbapenems in preference to the others. However, meropenem MICs are generally lower than imipenem MICs for Gram-negatives-in particular for $P$. aeruginosa (but not necessarily $A$. baumannii)-and this, together with the good safety profile even at higher doses, may favour meropenem. Differences in the potential for selection of resistance reflect subtle variations in the mode of penetration and other characteristics. The clinical relevance of such differences between imipenem and meropenem is not clear. There is little, if any, experience with doripenem dosing regimens beyond $3 \times 1 \mathrm{~g}$ daily. Thus, despite its slightly higher activity against $P$. aeruginosa there is no clear preference to its use. However, there is more experience with the use of meropenem in high-dose and prolonged infusion regimens, as well as concerns regarding the increased risk of seizures with high doses of imipenem. With respect to the use of prolonged or continuous infusion, there are some concerns regarding the stability of imipenem $[11,12]$. For a duration of administration of carbapenems beyond $3 \mathrm{~h}$ the stability of solutions at room temperature need to be considered and strictly controlled.

\section{Aminoglycosides}

As aminoglycoside use has declined in many settings, aminoglycoside resistance has dropped and sometimes aminoglycosides are still effective against some XDR strains [13,14]. Owing to lack of evidence supporting monotherapy in this special patient population, aminoglycosides should, in general, only be used in combination because of the risk of the development of resistance on therapy as well as efficacy concerns [13]. As aminoglycosides reach high concentrations in the urine, monotherapy appears to be an option in urinary tract infections (UTIs). Observational data suggest that these drugs may be superior to polymyxin B or tigecycline when strictly targeting carbapenem-resistant Klebsiella $\mathrm{sp}$. in the urine and have been successfully used in a limited number of patients with UTIs [15].

There are even less data on the appropriate dosage of aminoglycosides to be used in the treatment of XDR and PDR organisms. However, given the fact that they are concentrationdependent antibiotics and that septic patients might have augmented renal clearance of these drugs, the consensus was to use dosages on the high side of the dosing range. On the other hand, the duration of aminoglycoside therapy should be minimised to the safest level appropriate to reduce the risk of toxicity. PK/PD modelling suggests that a daily $10 \mathrm{mg} / \mathrm{kg}$ dose of gentamicin or tobramycin provides an $80 \%$ probability of response, even for an MIC of $4.0 \mu \mathrm{g} / \mathrm{mL}$, with a negligible likelihood of toxicity [16]. As stated before, the dosage regimen for the individual patient needs to ensure an adequate drug exposure in this patient in relation to the MIC and should be re-evaluated according to TDM [17].

\section{Colistin}

There has been marked renewed interest in this agent as, very often, XDR organisms are only susceptible to this agent. Colistin fell out of use because of concerns about nephrotoxicity when better tolerated antibiotics became available. In recent years, its use has increased markedly because of the growing incidence of XDR Enterobacteriaceae, $P$. aeruginosa and Acinetobacter spp. Despite the revival of this old drug and usage as a last-resort antibiotic, colistin has never been subjected to contemporary drug development procedures, and major gaps in our knowledge have only just recently been addressed [18]. Although this agent is reportedly effective in vitro against most strains of XDR Gram-negative bacterial infections, the actual clinical outcomes are often suboptimal, especially when given as a single drug $[19,20]$. This is partly due to the severe underlying diseases often associated with patients infected with XDR bacteria but is also caused by inadequate dosing regimens that have been employed empirically for more than 50 years. There are many factors that need to be taken into account in understanding the PK/PD properties of colistin, including its complex pharmacokinetics [21]. Colistin, a mixture of several compounds, is administered as the inactive prodrug colistimethate sodium (CMS). CMS and colistin have mixed routes of renal and non-renal elimination, a half-life that varies between different patient populations, and a volume of distribution that increases with critical illness. In addition, protein binding is concentration-dependent. The complex pharmacokinetics of colistin have only recently begun to unfold based on newly developed specific analytical methods. Based on the PK characteristics, a loading dose to achieve active colistin concentrations quickly and a maintenance dosing regimen that is based on the patient's kidney function have been proposed. The doses of CMS used for systemic infections in adults range widely. Based on recent PK studies, active concentrations at the site of infection can only be achieved with high dosages. Some recent regimens used 4.5 million International Units (MIU) twice a day or 3 MIU three times a day after a loading dose of 9 MIU as this might provide a balance between good clinical effect and acceptable toxicity. On the other hand, such high doses revive the earlier concerns regarding renal and neurological toxicity. There is clearly a need for randomised clinical trials to determine optimal dosing regimens of colistin in order to optimise clinical outcome, minimise the development of resistance, reduce toxicity and test optimum combinations.

Multicentre randomised studies, one funded by the 7 th Framework Program of the European Union (EU) (AIDA-Preserving Old Antibiotics for the Future) and one clinical study funded by the US National Institute of Allergy and Infectious Diseases (NIAID), are underway to compare colistin treatment with a combination regimen of colistin + carbapenem. Until more information is available, CMS should be exclusively given to patients with infections caused by XDR Gram-negative bacteria susceptible only to colistin. This drug should not be used to treat infections caused by bacteria susceptible to other antibiotics and should not be used as a single drug although clinical evidence is currently scarce [22].

Colistin has been used in a nebulised form for the prevention and treatment of respiratory tract infections, although the evidence for this indication outside the cystic fibrosis area is very limited [23]. Preliminary data in a small series of critically ill patients with ventilator-associated pneumonia (VAP) caused by carbapenem-resistant Acinetobacter and K. pneumoniae could not show superiority of a regimen that included concomitant inhalation of colistin in addition to intravenous (i.v.) treatment compared with i.v. treatment alone [24], although it was limited by small patient numbers and study design issues [25]. The results of small retrospective studies are conflicting, as demonstrated by other studies with improved survival with concomitant administration of aerosolised and i.v. colistin in patients with VAP [26]. Pooling the data of relevant clinical studies, adjunctive aerosolised colistin therapy did not reach statistically significant results 
compared with i.v. colistin alone [27]. More concrete clinical data are needed to support such treatment decisions.

Adjunct intrathecal colistin has been used for ventriculitis caused by XDR organisms but the evidence is largely confined to case reports [28]. A recent review of 83 episodes of MDR/XDR A. baumannii ventriculitis/meningitis concluded that intraventricular or intrathecal administration with concomitant i.v. colistin represents an important mode of last-resort therapy [29].

\section{Tigecycline}

There was some optimism initially that tigecycline retained activity against a portion of XDR Enterobacteriaceae and $A$. baumannii, but more recent studies showed only limited usefulness of tigecycline in CPE (47\% susceptible) with resistance scattered amongst $K$. pneumoniae and Enterobacter spp. [30]. Similarly, contemporary isolates from Greece were only susceptible in $64 \%$ of cases for XDR K. pneumoniae and $44 \%$ for A. baumannii [31]. Clinically, the use of tigecycline is limited due to lack of controlled studies targeting XDR organisms, concerns regarding efficacy and safety in critically ill patients, as well as the rapid development of resistance. The emergence of resistance via upregulation of efflux pumps during therapy is well described and a matter of concern [32]. The efficacy of tigecycline in clinical trials is related to low MICs, low baseline albumin concentration, lower severity and absence of VAP [33], usually not representing the patient population with XDR infection. Pooling non-inferiority studies with tigecycline showed excess mortality in critically ill patients in approved and non-approved indications, prompting a US Food and Drug Administration (FDA) warning against the usage of tigecycline when other options are available [34,35]. In addition, tigecycline is also hampered by low concentrations in the blood and protein binding of ca. 70\% that limits its use in bloodstream infections [36]. Increasing the dose of tigecycline is not generally recommended as it is poorly tolerated with increasing risk of nausea/vomiting related to higher drug exposure and the consequences of this are not well described [37]. Although tigecycline monotherapy results in higher failure rates [19,38] there is, however, some evidence that tigecycline in combination with colistin and a carbapenem could be effective in the treatment of severely ill patients with XDR Gram-negative infections. This remains to be shown in larger prospective studies.

\section{Fosfomycin}

Fosfomycin is active in vitro against some XDR Gram-negative bacteria [39] and could be a component of a combination regimen for the treatment of these infections. The concentrations required for activity are, however, relatively high for non-fermenters. There are only a few available data on PK/PD characteristics and clinical outcomes of fosfomycin $[40,41]$. Based on in vitro data and PK/PD modelling, a high dose and prolonged infusion combination strategy has been suggested [40]. Clinically, doses of $4 \mathrm{~g}$ four times daily and up to $8 \mathrm{~g}$ three times daily have been reported $[41,42]$, but appropriate dosage regimens in XDR situations have not yet been established.

There are concerns about fosfomycin's lack of efficacy for $A$. baumannii as well as rapid development of resistance in XDR Gram-negative bacteria (especially $P$. aeruginosa) during therapy even when used in combination $[43,44]$. Fosfomycin should never be used as monotherapy for severe systemic infections. As the i.v. form of fosfomycin is not approved in many countries, most experience is with oral preparations of the agent [42]. This achieves high concentrations in the urinary tract and has been used in the treatment of XDR and even PDR UTIs. Fosfomycin's limited availability as an i.v. formulation and missing PK/PD data as well as clinical information restricts its use in severely ill patients.

\section{Use of combination therapy}

Combination therapy as a strategy against XDR and PDR microorganisms is increasingly being used despite the paucity of clinical evidence $[45,46]$. In terms of susceptibility, it should be realised that susceptibility breakpoints are always based on monotherapy regimens and are therefore less suitable to use as a predictor for clinical outcome than usually thought. Exploring combination therapy, a recent retrospective multicentre study focusing on $K$. pneumoniae carbapenemase (KPC)-producing $K$. pneumoniae bloodstream infections showed that carbapenems could be effective in combination with other agents but this did not apply equally to organisms with an MIC $>8 \mu \mathrm{g} / \mathrm{mL}$ [47]. The authors concluded that combined treatment with two or more drugs with in vitro activity against the isolate, especially those also including a carbapenem, may be more effective than active monotherapy [47]. Similar results of a survival benefit with combination therapy including a carbapenem were reported by Qureshi et al. [38]. Tzouvelekis et al. compiled the results of 34 small studies involving a total of 298 patients with either KPC- or metallo- $\beta$-lactamase (MBL)-producing K. pneumoniae [19]. They found that the most effective regimens were those that included two active agents including a carbapenem. Combinations of carbapenem with colistin (5.5\% of failures) or with an aminoglycoside (6.2\%) performed significantly better than when these drugs were used alone or as part of other combinations, whilst combinations of tigecycline (24\% of failures), colistin (32\%) and aminoglycosides (33.3\%) in regimens not including a carbapenem exhibited higher failure rates. On the other hand, the use of tigecycline or colistin as single active agents resulted in higher failure rates $(35.7 \%$ and $47.2 \%$, respectively). The superiority of combination therapy is supported by another recent review on current clinical experience with carbapenemase-producing Gram-negative bacteria [48].

Clinical evidence for the superiority of combination therapy in XDR $P$. aeruginosa infections has not been established yet. The published meta-analysis did not stratify for the resistance status as it included mostly studies in the pre-XDR era [49-51]. Also, the study by Kumar et al. did not focus on XDR P. aeruginosa and does not provide answers to this clinical question [52]. As few therapy options exist for XDR $P$. aeruginosa [53]-usually colistin, less commonly fosfomycin and aminoglycosides-combination therapy appears to be preferable based on non-clinical data of the abovementioned antibiotics. Clinical data are warranted.

Acinetobacter spp. is increasingly becoming more XDR with few therapeutic options remaining. Similarly, there are no well designed clinical trials to compare treatment regimens for such infections. Most available data are derived from uncontrolled case series, animal models or in vitro studies. Investigations evaluating combinations of rifampicin, sulbactam, aminoglycosides, colistin, carbapenems and other drugs for the management of XDR Acinetobacter infections have shown conflicting data [54]. A lack of controlled clinical trials makes it difficult to evaluate the role of combination therapy for XDR Acinetobacter infection.

Because of the limited activity and rapid emergence of resistance to colistin, this drug is often propagated as a component of a combination as mentioned in the carbapenem and tigecycline sections. Based on the synergism between colistin and other antibiotics in vitro and in animal studies, a variety of combinations have been used in the clinical setting and included in uncontrolled studies or single case series. Besides the combinations of colistin with carbapenems or tigecycline, combination regimens of colistin + rifampicin have been widely discussed but the real clinical benefit still needs to be assessed [55]. 


\section{Novel antibiotics in development}

Most research and development (R\&D) efforts focus on the development of new analogues of well known antibacterial classes, although cross-resistance is already apparent or anticipated against every one of the new analogues currently in the development pipeline. Therefore, such modified analogues of existing antibiotic classes are less likely to be useful for XDR or PDR pathogens. Similarly, the concept of combining a known cephalosporin or carbapenem analogue with a new $\beta$-lactamase inhibitor (BLI) with extended or narrow $\beta$-lactamase coverage appears to buy some time in the resistance race. The long-known cephalosporin ceftazidime as well as the new cephalosporin analogues ceftaroline and ceftolozane are being developed in combination with the old BLI tazobactam or the new BLI avibactam. Avibactam expands the inhibitory spectrum of tazobactam to include KPC (class A), class $C$ and some OXA type $\beta$-lactamases but not the rapidly spreading metallo-enzymes (e.g. NDM). XDR Gramnegatives may have MBLs, upregulated efflux pumps, porin deficiencies or hyperproduction of extended-spectrum $\beta$-lactamases/carbapenemases among their resistance traits. All insurmountable hurdles for any BLI! In addition to avibactam, at least six more BLIs are in development, with considerable gaps in their activity that remain to be bridged. The phase 3-ready new aminoglycoside analogue plazomicin overcomes many widespread aminoglycoside-specific resistance mechanisms [56].

Only R\&D of new chemical entities unrelated to a known antibacterial scaffold, not showing cross-resistance to existing antibacterial drug classes and with a low propensity for emerging resistance, would be a promising option against XDR strains. However, very few such R\&D programmes are publicly disclosed and they are not enough to fill the R\&D pipelines to increase the likelihood of novel drugs eventually reaching the patient. Novel antibiotics without potential cross-resistance with established drugs and promising activity against XDR bacteria are neither on the horizon nor anticipated for the near future. Governments in the USA and EU have initiated several high-level initiatives including a wide variety of policies and incentives designed to jump start the development of novel antibacterial drugs and technologies, as recently reviewed by Theuretzbacher $[1,57]$.

\section{Conclusion}

Multiresistant Gram-negative bacilli are increasing threats worldwide. Whilst prevention, antibiotic stewardship and effective infection control strategies will be paramount in the efforts to control the emergence and spread of these organisms, novel agents are urgently needed to treat patients already infected with XDR and PDR organisms. Right now, we are primarily limited to combinations of existing agents including polymyxins, aminoglycosides, tigecycline, fosfomycin and carbapenems. The latter agents appear to retain some activity against carbapenemaseproducing organisms when used in combination and for organisms with MICs up to $8 \mu \mathrm{g} / \mathrm{mL}$ in the majority of patients when optimised dosing regimens are used. Until newer agents may be available for clinical use in the far future, the only option is to use existing agents in a better way, for instance by optimising exposures and the use of combination therapy [58]. High-quality randomised clinical trials would provide essential information to determine the best combination of established antibiotics that can be used to treat XDR and PDR Gram-negative infections. More research and practical guidance for individualising dosage regimens based on TDM and clinical decision support systems should be considered to achieve optimal exposure in critically ill patients. Presently, evidence-based recommendations on how to optimally treat critically ill patients with XDR or PDR Gram-negative organisms are not possible as clinical data are scarce or missing altogether. General principles of treatment, such as choosing the most appropriate antibiotics based on MIC data as well as considering the individual drug exposure based on TDM, would help to optimise therapy but may not be available in all institutions.

\section{Funding}

None.

\section{Competing interests}

PAT received research support from GSK, Sanofi-Pasteur, Inviragen, Fab'entech, Baxter and Adamas, and honoraria from MSD, Johnson and Johnson, $3 \mathrm{M}$ and AstraZeneca (last 5 years); GLH received consultancy/lecture fees from Janssen, MSD and Phoenix Argentina Laboratories; GLD has served as an advisory board member for Pfizer Hellas, Novartis, MSD and Achaogen and has also received a research grant from Pfizer Hellas; MEF participated in advisory boards of Achaogen, Astellas, AstraZeneca, Bayer, Basilea and Pfizer, received lecture honoraria from Angelini, Astellas, AstraZeneca, Cipla, Glenmark, Merck and Novartis, and received research support from Angelini, Astellas and Rokitan (last 10 years); TM received grants and support from Pfizer, Angelini, Astellas Pharma, Eli Lilly, Janssen-Cilag, Novartis, Sanofi-Aventis, Schering-Plough, Valeas, Wyeth Lederle and Zambon (last 5 years); AN received grants and support from Astra-Zeneca, Gilead, Janssen, MSD and Pfizer (last 2 years). All other authors declare no competing interests.

\section{Ethical approval}

Not required.

\section{References}

[1] Theuretzbacher U. Global antibacterial resistance: the never-ending story. Journal of Global Antimicrobial Resistance 2013. http://dx.doi.org/10.1016/ j.jgar.2013.03.010. in press.

[2] Magiorakos AP, Srinivasan A, Carey RB, Carmeli Y, Falagas ME, Giske CG, et al. Multidrug-resistant, extensively drug-resistant and pandrug-resistant bacteria: an international expert proposal for interim standard definitions for acquired resistance. Clinical Microbiology and Infection 2012;18:268-81.

[3] Poirel L, Bonnin RA, Nordmann P. Analysis of the resistome of a multidrugresistant NDM-1-producing Escherichia coli strain by high-throughput genome sequencing. Antimicrobial Agents and Chemotherapy 2011;55:4224-9.

[4] Hombach M, Bloemberg GV, Böttger EC. Effects of clinical breakpoint changes in CLSI guidelines 2010/2011 and EUCAST guidelines 2011 on antibiotic susceptibility test reporting of Gram-negative bacilli. Journal of Antimicrobial Chemotherapy 2012;67:622-32.

[5] Kahlmeter G, Brown DF, Goldstein FW, MacGowan AP, Mouton JW, Osterlund A, et al. European harmonization of MIC breakpoints for antimicrobial susceptibility testing of bacteria. Journal of Antimicrobial Chemotherapy 2003;52:145-8.

[6] Mouton JW, Brown DF, Apfalter P, Cantón R, Giske CG, Ivanova M, et al. The role of pharmacokinetics/pharmacodynamics in setting clinical MIC breakpoints: the EUCAST approach. Clinical Microbiology and Infection 2012;18:E37-45.

[7] Esterly JS, Wagner J, McLaughlin MM, Postelnick MJ, Qi C, Scheetz MH. Evaluation of clinical outcomes in patients with bloodstream infections due to Gram-negative bacteria according to carbapenem MIC stratification. Antimicrobial Agents and Chemotherapy 2012;56:4885-90.

[8] Daikos GL, Markogiannakis A. Carbapenemase-producing Klebsiella pneumoniae: (when) might we still consider treating with carbapenems? Clinical Microbiology and Infection 2011:17:1135-41.

[9] Kuti JL, Dandekar PK, Nightingale CH, Nicolau DP. Use of Monte Carlo simulation to design an optimized pharmacodynamic dosing strategy for meropenem. Journal of Clinical Pharmacology 2003;43:1116-23.

[10] Hayashi Y, Lipman J, Udy AA, Ng M, McWhinney B, Ungerer J, et al. $\beta$-Lactam therapeutic drug monitoring in the critically ill: optimising drug exposure in patients with fluctuating renal function and hypoalbuminaemia. International Journal of Antimicrobial Agents 2013:41:162-6.

[11] Keel RA, Sutherland CA, Crandon JL, Nicolau DP. Stability of doripenem, imipenem and meropenem at elevated room temperatures. International Journal of Antimicrobial Agents 2011;37:184-5. 
[12] Mouton JW, Vinks AA. Continuous infusion of $\beta$-lactams. Current Opinion in Critical Care 2007:13:598-606.

[13] Durante-Mangoni E, Grammatikos A, Utili R, Falagas ME. Do we still need the aminoglycosides? International Journal of Antimicrobial Agents 2009;33:201-5.

[14] Maraki S, Mavros MN, Kofteridis DP, Samonis G, Falagas ME. Epidemiology and antimicrobial sensitivities of 536 multi-drug-resistant Gram-negative bacilli isolated from patients treated on surgical wards. Surgical Infections (Larchmt) 2012;13:326-31.

[15] Satlin MJ, Kubin CJ, Blumenthal JS, Cohen AB, Furuya EY, Wilson SJ, et al. Comparative effectiveness of aminoglycosides, polymyxin $\mathrm{B}$, and tigecycline for clearance of carbapenem-resistant Klebsiella pneumoniae from urine. Antimicrobial Agents and Chemotherapy 2011;55:5893-9.

[16] Drusano GL, Louie A. Optimization of aminoglycoside therapy. Antimicrobial Agents and Chemotherapy 2011;55:2528-33.

[17] van Lent-Evers NA, Mathôt RA, Geus WP, van Hout BA, Vinks AA. Impact of goal-oriented and model-based clinical pharmacokinetic dosing of aminoglycosides on clinical outcome: a cost-effectiveness analysis. Therapeutic Drug Monitoring 1999;21:63-73.

[18] Bergen PJ, Landersdorfer CB, Lee HJ, Li J, Nation RL. 'Old' antibiotics for emerging multidrug-resistant bacteria. Current Opinion in Infectious Diseases 2012;25:626-33.

[19] Tzouvelekis LS, Markogiannakis A, Psichogiou M, Tassios PT, Daikos GL. Carbapenemases in Klebsiella pneumoniae and other Enterobacteriaceae: an evolving crisis of global dimensions. Clinical Microbiology Reviews 2012:25:682-707

[20] Paul M, Bishara J, Levcovich A, Chowers M, Goldberg E, Singer P, et al. Effectiveness and safety of colistin: prospective comparative cohort study. Journal of Antimicrobial Chemotherapy 2010;65:1019-27.

[21] Couet W, Grégoire N, Marchand S, Mimoz O. Colistin pharmacokinetics: the fog is lifting. Clinical Microbiology and Infection 2012;18:30-9.

[22] Deris ZZ, Yu HH, Davis K, Soon RL, Jacob J, Ku CK, et al. The combination of colistin and doripenem is synergistic against Klebsiella pneumoniae at multiple inocula and suppresses colistin resistance in an in vitro pharmacokinetic/ pharmacodynamic model. Antimicrobial Agents and Chemotherapy 2012;56:5103-12.

[23] Michalopoulos A, Papadakis E. Inhaled anti-infective agents: emphasis on colistin. Infection 2010;38:81-8.

[24] Kofteridis DP, Alexopoulou C, Valachis A, Maraki S, Dimopoulou D, Georgopoulos D, et al. Aerosolized plus intravenous colistin versus intravenous colistin alone for the treatment of ventilator-associated pneumonia: a matched-control study. Clinical Infectious Diseases 2010;51:1238-44.

[25] Kwa AL, Falagas ME, Michalopoulos A, Tam VH. Benefits of aerosolized colistin for ventilator-associated pneumonia: absence of proof versus proof of absence? Clinical Infectious Diseases 2011;52:1278-9.

[26] Arnold HM, Sawyer AM, Kollef MH. Use of adjunctive aerosolized antimicrobial therapy in the treatment of Pseudomonas aeruginosa and Acinetobacter baumannii ventilator-associated pneumonia. Respiratory Care 2012;57:1226-33.

[27] Florescu DF, Qiu F, McCartan MA, Mindru C, Fey PD, Kalil AC. What is the efficacy and safety of colistin for the treatment of ventilator-associated pneumonia? A systematic review and meta-regression. Clinical Infectious Diseases 2012;54:670-80.

[28] Khawcharoenporn T, Apisarnthanarak A, Mundy LM. Intrathecal colistin for drug-resistant Acinetobacter baumannii central nervous system infection: a case series and systematic review. Clinical Microbiology and Infection 2010;16:888-94.

[29] Karaiskos I, Galani L, Baziaka F, Giamarellou H. Intraventricular and intrathecal colistin as the last therapeutic resort for the treatment of multidrug-resistant and extensively drug-resistant Acinetobacter baumannii ventriculitis and meningitis: a literature review. International Journal of Antimicrobial Agents 2013. http://dx.doi.org/10.1016/j.ijantimicag.2013.02.006. pii:S0924-8579(13) $00054-\mathrm{X}$.

[30] Livermore DM, Warner M, Mushtaq S, Doumith M, Zhang J, Woodford N. What remains against carbapenem-resistant Enterobacteriaceae? Evaluation of chloramphenicol, ciprofloxacin, colistin, fosfomycin, minocycline, nitrofurantoin, temocillin and tigecycline. International Journal of Antimicrobial Agents 2011;37:415-9.

[31] Zarkotou O, Pournaras S, Altouvas G, Pitiriga V, Tziraki M, Mamali V, et al. Comparative evaluation of tigecycline susceptibility testing methods for expanded-spectrum cephalosporin- and carbapenem-resistant Gram-negative pathogens. Journal of Clinical Microbiology 2012;50:3747-50.

[32] Spanu T, De Angelis G, Cipriani M, Pedruzzi B, D'Inzeo T, Cataldo MA, et al. In vivo emergence of tigecycline resistance in multidrug-resistant Klebsiella pneumoniae and Escherichia coli. Antimicrobial Agents and Chemotherapy 2012;56:4516-8.

[33] Bhavnani SM, Rubino CM, Hammel JP, Forrest A, Dartois N, Cooper CA, et al Pharmacological and patient-specific response determinants in patients with hospital-acquired pneumonia treated with tigecycline. Antimicrobial Agents and Chemotherapy 2012;56:1065-72.

[34] Prasad P, Sun J, Danner RL, Natanson C. Excess deaths associated with tigecycline after approval based on noninferiority trials. Clinical Infectious Diseases 2012;54:1699-709.

[35] US Food and Drug Administration (FDA). FDA drug safety communication: increased risk of death with Tygacil (tigecycline) compared to other antibiotics used to treat similar infections. http://www.fda.gov/Drugs/DrugSafety/ ucm224370.htm [accessed March 2013].
[36] Van Wart SA, Owen JS, Ludwig EA, Meagher AK, Korth-Bradley JM, Cirincione BB. Population pharmacokinetics of tigecycline in patients with complicated intra-abdominal or skin and skin structure infections. Antimicrobial Agents and Chemotherapy 2006;50:3701-7.

[37] Rubino CM, Bhavnani SM, Forrest A, Dukart G, Dartois N, Cooper A, et al. Pharmacokinetics-pharmacodynamics of tigecycline in patients with community-acquired pneumonia. Antimicrobial Agents and Chemotherapy 2012;56:130-6.

[38] Qureshi ZA, Paterson DL, Potoski BA, Kilayko MC, Sandovsky G, Sordillo E, et al. Treatment outcome of bacteremia due to KPC-producing Klebsiella pneumoniae: superiority of combination antimicrobial regimens. Antimicrobial Agents and Chemotherapy 2012;56:2108-13.

[39] Endimiani A, Patel G, Hujer KM, Swaminathan M, Perez F, Rice LB, et al. In vitro

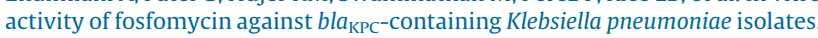
including those nonsusceptible to tigecycline and/or colistin. Antimicrobial Agents and Chemotherapy 2010;54:526-9.

[40] Asuphon O, Montakantikul P, Houngsaitong J, Sonthisombat P, Kiratisin P. Optimizing fosfomycin dosing for treatment of Pseudomonas aeruginosa infections in critically-ill patients based on pharmacokinetics/pharmacodynamics (PK/PD) target. In: 52nd Interscience Conference on Antimicrobial Agents and Chemotherapy (ICAAC). ASM Press; 2012. p. A-1279.

[41] Michalopoulos A, Virtzili S, Rafailidis P, Chalevelakis G, Damala M, Falagas ME. Intravenous fosfomycin for the treatment of nosocomial infections caused by carbapenem-resistant Klebsiella pneumoniae in critically ill patients: a prospective evaluation. Clinical Microbiology and Infection 2010;16:184-6.

[42] Falagas ME, Kastoris AC, Kapaskelis AM, Karageorgopoulos DE. Fosfomycin for the treatment of multidrug-resistant, including extended-spectrum $\beta$-lactamase producing, Enterobacteriaceae infections: a systematic review. Lancet Infectious Diseases 2010;10:43-50.

[43] Karageorgopoulos DE, Miriagou V, Tzouvelekis LS, Spyridopoulou K, Daikos GL. Emergence of resistance to fosfomycin used as adjunct therapy in KPC Klebsiella pneumoniae bacteraemia: report of three cases. Journal of Antimicrobial Chemotherapy 2012;67:2777-9.

[44] Karageorgopoulos DE, Wang R, Yu XH, Falagas ME. Fosfomycin: evaluation of the published evidence on the emergence of antimicrobial resistance in Gramnegative pathogens. Journal of Antimicrobial Chemotherapy 2012;67: 255-68.

[45] Abad CL, Kumar A, Safdar N. Antimicrobial therapy of sepsis and septic shockwhen are two drugs better than one? Critical Care Clinics 2011;27:e1-27.

[46] Paul M, Leibovici L. Combination antimicrobial treatment versus monotherapy: the contribution of meta-analyses. Infectious Disease Clinics of North America 2009;23:277-93.

[47] Tumbarello M, Viale P, Viscoli C, Trecarichi EM, Tumietto F, Marchese A, et al. Predictors of mortality in bloodstream infections caused by Klebsiella pneumoniae carbapenemase-producing K. pneumoniae: importance of combination therapy. Clinical Infectious Diseases 2012;55:943-50.

[48] Akova M, Daikos GL, Tzouvelekis L, Carmeli Y. Interventional strategies and current clinical experience with carbapenemase-producing Gram-negative bacteria. Clinical Microbiology and Infection 2012;18:439-48.

[49] Safdar N, Handelsman J, Maki DG. Does combination antimicrobial therapy reduce mortality in Gram-negative bacteraemia? A meta-analysis. Lancet Infectious Diseases 2004;4:519-27.

[50] Vardakas KZ, Tansarli GS, Bliziotis IA, Falagas ME. $\beta$-Lactam plus aminoglycoside or fluoroquinolone combination versus $\beta$-lactam monotherapy for Pseudomonas aeruginosa infections: a meta-analysis. International Journal of Antimicrobial Agents 2013;41:301-10.

[51] Paul M, Benuri-Silbiger I, Soares-Weiser K, Leibovici L. $\beta$ lactam monotherapy versus $\beta$ lactam-aminoglycoside combination therapy for sepsis in immunocompetent patients: systematic review and meta-analysis of randomised trials. British Medical Journal 2004;328:668.

[52] Kumar A, Zarychanski R, Light B, Parrillo J, Maki D, Simon D, et al. Cooperative Antimicrobial Therapy of Septic Shock (CATSS) Database Research Group. Early combination antibiotic therapy yields improved survival compared with monotherapy in septic shock: a propensity-matched analysis. Critical Care Medicine 2010;38:1773-85.

[53] Tam VH, Chang KT, Abdelraouf K, Brioso CG, Ameka M, McCaskey LA, et al. Prevalence, resistance mechanisms, and susceptibility of multidrug-resistant bloodstream isolates of Pseudomonas aeruginosa. Antimicrobial Agents and Chemotherapy 2010;54:1160-4.

[54] Maragakis LL, Perl TM. Acinetobacter baumannii: epidemiology, antimicrobial resistance, and treatment options. Clinical Infectious Diseases 2008;46 1254-63.

[55] Drapeau CM, Grilli E, Petrosillo N. Rifampicin combined regimens for Gramnegative infections: data from the literature. International Journal of Antimicrobial Agents 2010;35:39-44.

[56] Zhanel GG, Lawson CD, Zelenitsky S, Findlay B, Schweizer F, Adam H, et al. Comparison of the next-generation aminoglycoside plazomicin to gentamicin, tobramycin and amikacin. Expert Review of Anti-infective Therapy 2012:10:459-73.

[57] Theuretzbacher U. Accelerating resistance, inadequate antibacterial drug pipelines and international responses. International Journal of Antimicrobial Agents 2012;39:295-9.

[58] Mouton JW, Ambrose PG, Canton R, Drusano GL, Harbarth S, MacGowan A, et al. Conserving antibiotics for the future: new ways to use old and new drugs from a pharmacokinetic and pharmacodynamic perspective. Drug Resistance Updates 2011;14:107-17. 